PROCEEDINGS OF THE

AMERICAN MATHEMATICAL SOCIETY

Volume 133, Number 6, Pages 1723-1726

S 0002-9939(04)07672-5

Article electronically published on December 20, 2004

\title{
ESSENTIAL NUMERICAL RANGE OF ELEMENTARY OPERATORS
}

\author{
M. BARRAA
}

(Communicated by Joseph A. Ball)

\begin{abstract}
Let $A=\left(A_{1}, \ldots, A_{p}\right)$ and $B=\left(B_{1}, \ldots, B_{p}\right)$ denote two $p$-tuples of operators with $A_{i} \in \mathcal{L}(H)$ and $B_{i} \in \mathcal{L}(K)$. Let $R_{2, A, B}$ denote the elementary operators defined on the Hilbert-Schmidt class $\mathcal{C}^{2}(H, K)$ by $R_{2, A, B}(X)=$ $A_{1} X B_{1}+\ldots+A_{p} X B_{p}$. We show that$$
c o\left[\left(W_{e}(A) \circ W(B)\right) \cup\left(W(A) \circ W_{e}(B)\right)\right] \subseteq V_{e}\left(R_{2, A, B}\right) .
$$

Here $V_{e}($.$) is the essential numerical range, W($.$) is the joint numerical range$ and $W_{e}($.$) is the joint essential numerical range.$

\section{Introduction}

Let $\mathcal{L}(H)$ denote the algebra of all bounded linear operators on a separable infinite-dimensional Hilbert space $H$. Let $A=\left(A_{1}, \ldots, A_{p}\right)$ and $B=\left(B_{1}, \ldots, B_{p}\right)$ denote two $p$-tuples of operators with $A_{i} \in \mathcal{L}(H)$ and $B_{i} \in \mathcal{L}(K)$. Let $R_{A, B}$ : $\mathcal{L}(H) \longrightarrow \mathcal{L}(H)$ denote the elementary operators defined by

$$
R_{A, B}(X)=A_{1} X B_{1}+\ldots+A_{p} X B_{p} .
$$

The class of Hilbert-Schmidt operators from a Hilbert space $H$ to a Hilbert space $K$ will be denoted by $\mathcal{C}^{2}(H, K)$ and, of course, $\mathcal{C}^{2}(H)=\mathcal{C}^{2}(H, H)$; see [8]. Recall that $\mathcal{C}^{2}(H, K)$ is a Hilbert space and that $A_{i} X B_{i} \in \mathcal{C}^{2}(H, K)$ for every $A_{i} \in \mathcal{L}(H), X \in \mathcal{C}^{2}(H, K)$ and $B_{i} \in \mathcal{L}(K)$. So the elementary operator $R_{A, B}$ is a bounded endomorphism of $\mathcal{C}^{2}(H, K)$. We denote by $R_{2, A, B}$ the restriction of $R_{A, B}$ to $\mathcal{C}^{2}(H, K)$.

If $\mathcal{A}$ is a Banach algebra with unit $e$, the algebraic numerical range of an arbitrary element $a \in \mathcal{A}$ is defined by

$$
V(a)=\left\{f(a) ; f \in \mathcal{A}^{\prime},\|f\|=f(e)=1\right\} .
$$

Here, of course, $\mathcal{A}^{\prime}$ denotes the space of all continuous linear functionals on $\mathcal{A}$. Recall that $V(a)$ is a compact convex set.

For $T \in \mathcal{L}(H)$, the numerical range of $T$ is defined as

$$
W(T)=\{\langle T x, x\rangle:\|x\|=1\} .
$$

Received by the editors November 14, 2003 and, in revised form, February 13, 2004.

2000 Mathematics Subject Classification. Primary 47B47; Secondary 47A12.

Key words and phrases. Elementary operators, essential numerical range, Hilbert-Schmidt class.

(C)2004 American Mathematical Society Reverts to public domain 28 years from publication 
The essential numerical range, $V_{e}(T)$, is (by definition) the numerical range of the coset $T+K(H)$ in the Calkin algebra $\mathcal{L}(H) / K(H)$ where $K(H)$ is the ideal of all compact operators on $H$; see [1] 2] and [9].

It is known that $V_{e}(T) \subseteq W(T)^{-}$, the closure of $W(T)$.

For a $p$-tuple $A=\left(A_{1}, \ldots, A_{p}\right)$ of operators on a Hilbert space $H$ we define:

- i) the joint numerical range of $A$ by

$$
W(A)=\left\{\left(\left\langle A_{1} x, x\right\rangle, \ldots,\left\langle A_{p} x, x\right\rangle\right) ; \quad x \in H,\|x\|=1\right\} ;
$$

- ii) the joint essential numerical range of $A$ by $\lambda \in W_{e}(A)$ if $\lambda=\left(\lambda_{1}, \ldots, \lambda_{p}\right) \in C^{p}$ and there exists an orthonormal sequence $\left(x_{n}\right)$ in $H$ such that $\lambda_{i}=\operatorname{Lim}\left\langle A_{i} x_{n}, x_{n}\right\rangle, i=1, \ldots, p$.

To simplify the statements, we shall use the following notation: for $\alpha, \beta \in C^{n}$, we let $\alpha \circ \beta=\sum_{i=1}^{p} \alpha_{i} \beta_{i}$ and for $K, L \subseteq C^{n}$,

$$
K \circ L=\{\alpha \circ \beta, \quad \alpha \in K, \beta \in L\} .
$$

For vectors $x, y \in H$, the notation $x \otimes y$ will refer to the operator in $L(H)$ defined by $x \otimes y(z)=\langle z, y\rangle . x$.

In the past, elementary operators and their restrictions to norm ideals in $L(H)$ have been studied by many authors. Up to now, their spectra and their essential spectra have been characterized; see [4, 5, 3]. In [7], B. Magajna has determined the essential numerical range of the restriction of a generalized derivation to the class of Hilbert-Schmidt.

In this paper, we give some results about the essential numerical range of the restriction of an elementary operator to the class of Hilbert-Schmidt. More precisely, we prove that

$$
c o\left[\left(W_{e}(A) \circ W(B)\right) \cup\left(W(A) \circ W_{e}(B)\right)\right] \subseteq V_{e}\left(R_{2, A, B}\right),
$$

and we give some consequences of this inclusion.

\section{The ESSEntial NUMERICAL RANGe}

We need the following characterization of the essential numerical range, obtained by Fillmore, Stampfli, and Williams in [9].

Lemma 2.1. Let $T \in \mathcal{L}(H)$. Each of the following conditions is necessary and sufficient in order that $\lambda \in V_{e}(T)$ :

(1) $\left\langle T x_{n}, x_{n}\right\rangle \longrightarrow \lambda$ for some sequence $\left(x_{n}\right)$ of unit vectors such that $x_{n} \rightarrow 0$ weakly.

(2) $\left\langle T e_{n}, e_{n}\right\rangle \longrightarrow \lambda$ for some orthonormal sequence $\left(e_{n}\right)$.

The main result of this paper is the following.

Theorem 2.2. Let $H, K$ be two separable Hilbert spaces and $A=\left(A_{1}, \ldots, A_{p}\right)$, $B=\left(B_{1}, \ldots, B_{p}\right)$ two $p$-tuples with $A_{i} \in \mathcal{L}(H)$ and $B_{i} \in \mathcal{L}(K)$ for $i=1, \ldots, p$. Then

$$
\text { co }\left[\left(W_{e}(A) \circ W(B)\right) \cup\left(W(A) \circ W_{e}(B)\right)\right] \subseteq V_{e}\left(R_{2, A, B}\right) .
$$

Proof. Let $\lambda \in W_{e}(A)$. There exists an orthonormal sequence $\left(x_{n}\right)$ in $H$ such that

$$
\lambda_{i}=\operatorname{Lim}\left\langle A_{i} x_{n}, x_{n}\right\rangle \text { for each } i=1, \ldots, p .
$$

Let $\mu \in W(B)$. There exists a unit vector $y$ in $K$ such that $\mu_{i}=\left\langle B_{i} y, y\right\rangle$. It is easily verified that $\left(x_{n} \otimes y\right)$ is an orthonormal sequence in $C^{2}(H, K)$ and

$$
\left\langle A_{i}\left(x_{n} \otimes y\right) B_{i}, x_{n} \otimes y\right\rangle=\operatorname{tr}\left(A_{i}\left(x_{n} \otimes y\right) B_{i}\left(y \otimes x_{n}\right)\right)=\left\langle A_{i} x_{n}, x_{n}\right\rangle \cdot\left\langle B_{i} y, y\right\rangle .
$$


Hence,

$$
\left\langle R_{2, A, B}\left(x_{n} \otimes y\right), x_{n} \otimes y\right\rangle=\sum_{i=1}^{p}\left\langle A_{i} x_{n}, x_{n}\right\rangle \cdot\left\langle B_{i} y, y\right\rangle .
$$

That is, $\lambda \circ \mu \in V_{e}\left(R_{2, A, B}\right)$.

The essential numerical range of the restriction of a generalized derivation to the class of Hilbert-Schmidt has been computed in [7, by B. Magajna. He has shown that

$$
V_{e}\left(\delta_{2, A, B}\right)=c o\left[\left(V_{e}(A)-W(B)^{-}\right) \cup\left(W(A)^{-}-V_{e}(B)\right)\right]
$$

Here we give only the easiest inclusion.

Corollary 2.3. For $A \in \mathcal{L}(H)$ and $B \in \mathcal{L}(K)$,

$$
c o\left[\left(V_{e}(A)-W(B)^{-}\right) \cup\left(W(A)^{-}-V_{e}(B)\right)\right] \subseteq V_{e}\left(\delta_{2, A, B}\right) .
$$

If, in addition, $V_{e}(A)=W(A)^{-}$or $V_{e}(B)=W(B)^{-}$, then we have equality.

Corollary 2.4. For $A \in \mathcal{L}(H)$ and $B \in \mathcal{L}(K)$,

$$
\begin{gathered}
c o\left[\left(V_{e}(A) \cdot W(B)^{-}\right) \cup\left(W(A)^{-} \cdot V_{e}(B)\right)\right] \subseteq V_{e}\left(M_{2, A, B}\right), \\
V_{e}\left(L_{2, A}\right)=W(A)^{-} \quad \text { and } \quad V_{e}\left(R_{2, B}\right)=W(B)^{-} .
\end{gathered}
$$

Proof. We have $W(A)^{-} \subseteq V_{e}\left(L_{2, A}\right) \subseteq W\left(L_{2, A}\right)^{-}=W(A)^{-}$.

\section{NonNeGATIVE OPERATORS AND THE ESSENTIAL NUMERICAL RANGE}

Lemma 3.1. Let $A$ be a nonnegative, selfadjoint operator and $A B=B A$. Then

$$
V_{e}(A B) \subseteq V_{e}(A) V_{e}(B) .
$$

Proof. Let $\lambda \in V_{e}(A B)$. There exists a sequence $\left(x_{n}\right)$ of unit vectors in $H$ such that $x_{n} \rightarrow 0$ weakly and

$$
\lambda=\operatorname{Lim}\left\langle A B\left(x_{n}\right), x_{n}\right\rangle .
$$

Let $y_{n}=A^{\frac{1}{2}} x_{n}$. If $y_{n_{k}}=0$ for some subsequence, then 0 is in both sides of (1). If not and by passing to a subsequence if necessary, we can assume that $y_{n} \neq 0 \quad \forall n$. Put $z_{n}=\frac{y_{n}}{\left\|y_{n}\right\|}$. Then $\left(z_{n}\right)$ is a sequence of unit vectors with $z_{n} \rightarrow 0$ weakly and

$$
\lambda=\operatorname{Lim}\left\langle B z_{n}, z_{n}\right\rangle \cdot\left\langle A x_{n}, x_{n}\right\rangle .
$$

But $\operatorname{Lim}\left\langle B z_{n}, z_{n}\right\rangle \in V_{e}(B)$. So $\lambda \in V_{e}(A) V_{e}(B)$.

Corollary 3.2. Let $A \in \mathcal{L}(H)$ be a nonnegative, selfadjoint operator and $B \in$ $\mathcal{L}(K)$. Then

$$
V_{e}\left(M_{2, A, B}\right) \subseteq W(A)^{-} W(B)^{-} .
$$

Proof. Recall that $L_{2, A} R_{2, B}=R_{2, B} L_{2, A}, V_{e}\left(L_{2, A}\right)=W(A)^{-}$and $V_{e}\left(R_{2, B}\right)=$ $W(B)^{-}$. The rest is from Lemma 3.1 .

\section{ACKNOWLEDGEMENT}

The author wishes to express his thanks to the referee for several helpful comments concerning this paper. 


\section{REFERENCES}

1. F.F. Bonsall and J. Duncan, Numerical Ranges of Operators on Normed Spaces and Elements of Normed Algebras, London Math. Soc. Lecture Note Series 2, Cambridge, 1971. MR 0288583 (44:5779)

2. F.F Bonsall and J. Duncan, Numerical ranges, vol II, Cambridge University Press (1973). MR0442682 (56:1063)

3. R. Curto, The spectra of elementary operators, Indiana Univ. Math.J., 32(1983), 193-197. MR0690184 (84e:47005)

4. L.A. Fialkow, $A$ note on norm ideals and the operator $X \rightarrow A X-X B$, Israel J. Math., 32(1979), 331-348. MR0571087|(81g:47046)

5. L. A. Fialkow, Spectral properties of elementary operators, Acta Sci. Math., 46(1983), 269282. MR0739043 (85h:47003)

6. C. K. Fong, Normal operators on Banach space, Glasgow Math. J. 20(1979), 163-168. MR0536389 (80e:47020)

7. B. Magajna, On the essential numerical range of a generalized derivation, Proc. Amer. Math. Soc., 99(1987), 86-92. MR0866435 (88e:47065)

8. R. Schatten, Norm ideals of completely continuous operators, Ergebnisse der Math. und ihrer Grenzgebiete, 27, 2nd ed., Springer-Verlag, Berlin-Heidelberg-New York,1970. MR0257800 (41:2449)

9. J. Stampfli, P. Fillmore and J. Williams, On the essential numerical range, the essential spectrum and a problem of Halmos, Acta Sci. Math., 33(1973), 172-192. MR0322534(48:896)

Département de Mathématiques, Faculté des Sciences Semlalia, Marrakech, Maroc

E-mail address: barraa@ucam.ac.ma 